\title{
ACTION DE LA PHOTOPERIODE SUR LE CYCLE BIOLOGIQUE ET LA DIAPAUSE D'ANABOLIA NERVOSA CURTIS (TRICHOPTERA - LIMNEPHILIDAE)
}

\author{
par C. DENIS ${ }^{1}$
}

Des élevages réalisés à différentes photopériodes de 24 heures maintenues constantes pendant tout le développement montrent que :

- sous photophase moyenne (10-12 h) les larves entrent en diapause et y demeurent indéfiniment :

- sous photophase longue $(\geqslant 16 \mathrm{~h})$ ou courte $(<8 \mathrm{~h})$ les animaux se métamorphosent directement ou après une courte diapause.

La reprise du développement des animaux conditionnés à subir une diapause illimitée est obtenue par l'action d'une photopériode à photophase longue $(\geqslant 15 \mathrm{~h})$ qui doit durer une douzaine de jours au minimum et se prolonger pendans 2 mois pour que tous les animaux puissent se métamorphoser. Une photopériode à photophase courte $(<8 \mathrm{~h})$ provoque la rupture de la diapause avec une efficacité moindre. Cette rupture est plus aisée pour les $\&$ que pour les $q$.

Les résultats expérimentaux qui concordent avec les observations de terrain expliquent la présence de rares adultes en plein été.

\section{The effects of different photoperiods on the biological cycle and diapause of Anabolia nervosa Curtis (Trichoptera - Limnephilidae).}

Larvae were reared under different photoperiods of 24 hours which were kept constant throughout development. These experiments showed that:

- under a middle photophase (10-12 hours) all larvae entered diapause and remained in this state indefinitely;

- under a long ( $\geqslant 16$ hours) or a short $(<8$ hours) photophase, larvae metamorphosed either directly or after a limited diapause.

The resumption of development of animals conditioned to undergo an unlimited diapause was brought about by a long photophase ( $\geqslant 15$ hours) who must go on for about 12 days and be prolonged up to two months before all larvae metamorphosed.

A photoperiod with a short photophase $(<8$ hours) could also break diapause but its effectiveness was much less. This breaking was brought about more readily in males than in females.

The experimental results that agree with findings in the field give an explanation for the rare occurences of adults in summer.

1. Laboratoire de Biologie Animale $1^{\text {er }}$ Cycle, Université de Rennes I. Avenue du Général-Leclerc, 35042 Rennes Cedex. 


\section{1. - INTRODUCTION}

Les cycles d'Anabolia nervosa Curtis et d'Anabolia furcata Brauer sont connus dans leurs grandes lignes par les travaux de Hanna (1957) pour la première espèce et de Novak (1960) pour la seconde. Ces cycles commencent en automne. Les larves passent par cinq stades. La croissance larvaire, très faible en hiver, est forte au printemps. Elle se termine en juin pour $A$. furcata et les larves entrent alors en diapause. $A$. nervosa se comporte de la même façon, bien que Hanna ne le mentionne pas. Selon Novak, la diapause serait due aux longues périodes diurnes estivales et serait rompue par suite d'une diminution de la durée du jour à la fin de l'été. La nymphose commence en août ou en septembre et dure environ trois semaines. La période de vol des adultes se situe en septembre et octobre.

Cependant d'autres auteurs ont capturé des adultes des deux espèces en plein été, ce qui ne concorderait pas avec l'existence d'une diapause estivale. Ainsi Botosaneanu (1957) situe entre début juillet et fin septembre la période de vol de $A$. furcata en Roumanie. De même A. nervosa est signalé en France dès juillet par Killington (1930). Mais les renseignements les plus précis concernant la période de vol de A. nervosa ont été recueillis en Grande-Bretagne. Nous les devons au $\mathrm{D}^{\mathrm{r}}$ Crichton qui a eu la grande amabilité de nous communiquer les données des autres auteurs, et surtout les résultats inédits de ses propres travaux, résultats qu'il nous a autorisé à publier. Ainsi Baker (Crichton et Baker 1959) a capturé de 19-VII-1958 un adulte à Woolhampton près de Reading. BRINDLE (1965) signale la présence d'adultes, sans précision de nombre, en juin et juillet 1965 au Nord de l'Angleterre. Mais surtout Crichton a effectué des captures régulières à l'aide de pièges lumineux de 1964 à 1978. Il a rassemblé ses données dans trois diagrammes que nous présentons dans la figure 1. Les diagrammes montrent nettement que la période de vol se situe en septembre et octobre, avec un maximum d'individus capturés au début d'octobre. De plus ces diagrammes indiquent clairement que l'apparition d'adultes en été est rarissime. Il faut ajouter que le seul individu capturé en juillet (nuit des 27-28-VII-1970) était un mâle, de même que celui pris fin août (nuit des 24-25-VIII-1967).

Des expériences en laboratoire ont par ailleurs été effectuées sur A. nervosa (Denis 1974 et 1978). Elles ont montré que :

1) Lorsque le développement a lieu en hiver, ou sous un éclairement journalier de 12 heures, les animaux entrent tous en diapause, et ceci, quelle que soit la photopériode à la fin de la croissance. Les longues périodes diurnes des jours d'été ne représentent donc pas, comme le pensait Novak, le facteur responsable du déclenchement et du maintien de la diapause, du moins pour $A$. nervosa. 


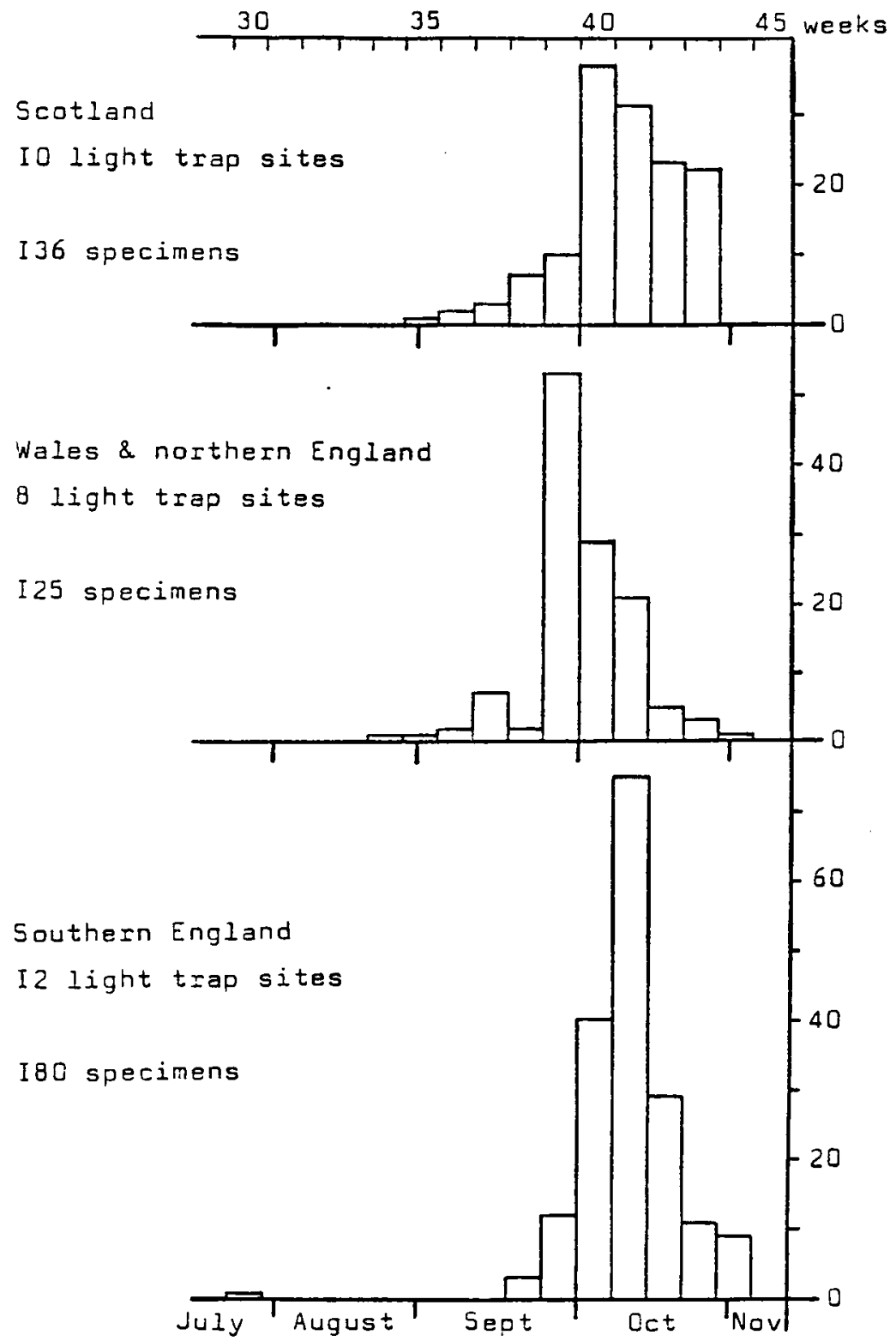

FIg. 1. - Diagrammes illustrant la répartition des captures d'adultes d'Anabolia nervosa par piégeage lumineux dans trois régions de Grande-Bretagne de 1964 à 1978. Travaux du $D^{r}$ Crichton grâce à l'aide du Rothamsted Insect Survey. Les captures sont comptabilisées pour l'ensemble des années, semaine par semaine, dans un système hebdomadaire fixe établi par le Rothamsted Insect Survey. 
2) Par contre lorsque le développement a lieu en été, ou sous un éclairement journalier de 18 heures, les animaux se métamorphosent directement ou subissent seulement une courte diapause.

3) Le conditionnement de la diapause est complexe. Cette dernière est induite par l'action d'une photopériode de type hivernal sur le troisième stade larvaire principalement.

Dans le but de mieux connaître l'action de la photopériode sur Anabolia nervosa et de pouvoir interpréter l'ensemble des données concernant cette espèce, nous avons cherché à préciser les points suivants :

- le rôle de différentes photopériodes sur le développement larvaire et la diapause.

- l'effet de changements de photopériode pendant la diapause.

Enfin l'interprétation des résultats de nos expériences n'aurait pas été complète sans une bonne connaissance de la répartition des entrées en nymphose et des émergences dans la nature. Nous présenterons donc, au début de ce travail, des données concernant ces deux points particuliers.

\section{2. - MATERIEL ET METHODES}

L'espèce Anabolia nervosa Curtis est commune en Bretagne. Les expériences ont été réalisées avec des larves issues de pontes obtenues en laboratoire; mais les animaux qui ont fourni ces pontes ont été récoltés à l'état de larves en diapause - en fin mai et début juin — et ayant donc effectué tout leur développement dans la nature.

La diapause se situe entre la fin de la croissance larvaire et la métamorphose. Les larves qui effectuent tout leur développement dans un fourreau mobile demeurent, durant la diapause, à l'intérieur de leur étui qu'elles ont préalablement fixé à un support. Ne possédant pas encore de critères précis pour situer exactement le début et la fin de la diapause, nous considérons qu'elle commence lorsque les larves cessent de s'alimenter et qu'elle se termine lorsqu'elles redeviennent actives et obturent les deux extrémités de leur fourreau, juste avant la nymphose.

Dans nos expériences, nous avons toujours utilisé des cycles photopériodiques de 24 heures. Dans le texte, pour caractériser les photopériodes, nous indiquerons la durée de la période diurne. Mais nous ne considérons pas, pour autant, que les animaux réagissent à la photophase (période diurne) plutôt qu'à la scotophase (période nocturne).

Les bacs d'élevage utilisés pour les expériences avaient une contenance de cinq litres. 
Toutes les expériences ont été faites à la température de $15^{\circ} \mathrm{C}$.

L'alimentation des larves était assurée par du ray-gras et des feuilles mortes.

\section{3. - EPOQUE ET REPARTITION DES ENTREES EN NYMPHOSE ET DES EMERGENCES DANS LA NATURE}

Ayant récolté pendant plusieurs années des larves en diapause que nous avons maintenues en laboratoire à la lumière du jour et à une température de 15 à $18{ }^{\circ} \mathrm{C}$ - conditions comparables à celles de la nature - nous avons noté à chaque fois les dates des entrées en nymphose et des émergences de ces animaux. Nous présentons, à titre d'exemple, dans la figure 2, les données concernant 73 larves récoltées en début juin 1978 .

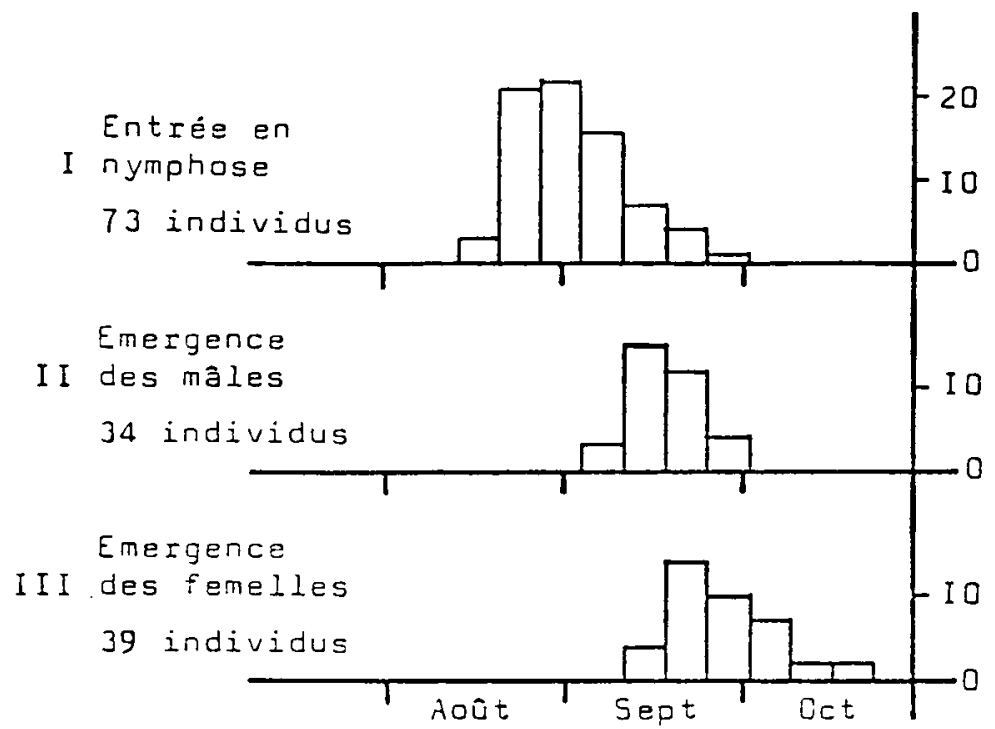

Fig. 2. - Diagrammes illustrant la répartition des entrées en nymphose et des émergences des femelles d'une population d'Anabolia nervosa dont le développement larvaire s'est déroulé dans la nature, en Bretagne et dont la métamorphose a eu lieu en laboratoire, à la lumière du jour et à une température de $15-18{ }^{\circ} \mathrm{C}$.

L'alimentation et la croissance larvaires se terminant durant la seconde quinzaine de mai ou début juin et le diagramme I montrant que les entrées en nymphose s'échelonnent de mi-août à fin septembre, la diapause dure donc de trois à quatre mois dans la nature. 
Le diagramme I montre également que les entrées en nymphose se produisent principalement pendant les derniers jours d'août et les premiers jours de septembre.

La comparaison des diagrammes II et III indique que la majorité des mâles émerge un peu plus tôt que la majorité des femelles. Nous avons en outre toujours remarqué que les premiers adultes qui apparaissent étaient des mâles et les derniers des femelles. Ces observations vont dans le sens de celles de Crichton et concordent avec celles de Novak (1960) pour A. furcata.

Le maximum des captures de Crichton se situe au début d'octobre. Or nos diagrammes montrent que la plupant des adultes émerge vers la mi-septembre. Il sera intéressant de chercher les raisons de ces ścarts.

\section{4. - ROLE DE LA PHOTOPERIODE SUR LE DEVELOPPEMENT LARVAIRE}

Des lots de 25 larves ont été élevés à différentes photopériodes maintenues constantes depuis l'éclosion jusqu'à la métamorphose ou la mort. Ces expériences ont été réalisées avec des photophases comprises entre 6 et 18 heures et échelonnées toutes lles deux heures.

\subsection{Résultats}

Dans tous les cas, la croissance larvaire a duré quatre mois ou presque : environ deux mois pour l'ensemble des quatre premiers stades et entre un mois et demi et deux mois pour la période d'alimentation et de croissance au cinquième et dernier stade larvaire. Dans nos expériences, la croissance des animaux a été beaucoup plus rapide que dans la nature en raison de l'abondance de la nourriture fournie et de la température relativement élevée.

Mais après la fin de leur croissance, les larves se sont comportées différemment selon les cas (voir tableau I).

Dans les lots maintenus sous photophase moyenne (10 et 12 heures) aucun animal ne s'est métamorphosé. Dans les autres lots, une partie des animaux, ou tous, sont entrés en nymphose, mais après des délais variables.

Pour les deux lots à 16 et à 18 heures, la mortalité est très faible (quatre larves mortes à la fin de la croissance ou peu après); nous pouvons donc considérer que ces conditions permettent la métamorphose de l'ensemble des animaux.

Dans les lots à 6 heures et à 14 heures, respectivement sept et huit 
Tableau I. - Réactions, à la fin de la vie larvaire, d'animaux maintenus depuis l'éclosion sous des photopériodes constantes. Chaque expérience porte sur 25 animaux. Les délais ne sont pas indiqués avec précision car il était impossible de déterminer au jour près la fin de l'alimentation de chaque animal.

Photophase Délai entre la fin de la croissance larvaire et l'entrée en nymphose.

$6 \mathrm{~h} \quad$ Une dizaine de jours à 3 mois pour 11 mâles et 7 femelles.

Et 7 animaux morts à l'état larvaire, les derniers après 5 mois.

$8 \mathrm{~h} \quad 2$ à 3 mois pour 3 mâles et 1 femelle. Ensuite, arrêt accidentel de l'expérience.

$10 \mathrm{~h}$ Aucune nymphose.

$12 \mathrm{~h}$ Tous les animaux sont morts à l'état larvaire après 4 à 7 mois.

$14 \mathrm{~h} \quad 2$ à 4 mois et demi pour 10 mâles et 6 femelles.

Et 9 animaux morts à l'état larvaire, les derniers après 5 mois.

$16 \mathrm{~h}$ Une dizaine de jours à 2 mois pour 10 mâles et 14 femelles. Et une larve morte entre-temps.

$18 \mathrm{~h}$ Une dizaine de jours à 2 mois pour 13 mâles et 9 femelles. Et 3 larves mortes entre-temps.

animaux sont morts à l'état larvaire, et presque tous entre trois et cinq mois après la fin de la croissance. Nous pouvons alors penser que la plupart de ces animaux étaient incapables d'entrer en nymphose. L'expérience sous photophase de 8 heures ayant été interrompue par une panne d'appareillage, nous ne savons pas ce que seraient devenus les animaux du lot demeurés à l'état larvaire.

Dans tous les lots où des animaux se sont métamorphosés, les premiers individus qui rompaient leur diapause étaient des mâles. Et d'une manière générale, la majorité de ces derniers entrait toujours en nymphose avant la majorité des femelles.

Dans les expériences sous photophase longue (16-18 heures) et sous photophase courte ( 6 heures), le délai entre la fin de la croissance et la métamorphose, court pour quelques individus mais échelonné sur deux ou trois mois pour l'ensemble de chaque population, conduit à penser que certains animaux ont eu un développement direct, tandis que les autres ont subi une diapause. Cette question a déjà été étudiée dans un précédent travail (Denis 1978). Les individus mâles qui entrent en nymphose les premiers après un délai inférieur à un mois ont, au tout début de la prénymphose, des testicules en pleine activité dont les lobes sont remplis de cystes en cours de spermatogènèse et de faisceaux de spermatozoïdes. Au contraire les mâles qui entrent en nymphose plus tardivement ont, au début de la prénymphose, des testicules au repos où quelques faisceaux de spermatozoïdes déjà formés sont tassés à la base des lobes, tandis que l'apex de ceux-ci est occupé par des cystes à spermatogonies et que leur zone centrale est vide. Ce dernier aspect du testicule est caractéristique pendant la diapause (Le Lannic 1976). En dehors de preuves fournies par l'étude 
ciu système endocrinien, nous pouvons donc penser, en nous référant aux mâles, que lorsque les animaux se développent sous photophase longue ou sous photophase courte, une partie d'entre eux présente un développement direct, tandis que les autres subissent une diapause.

\subsection{Conclusions}

La photopériode ne semble pas influencer la durée de la période d'alimentation et de croissance des larves, mais elle exerce une action importante à la fin du cinquième stade. Les résultats de nos expériences et ceux de notre étude antérieure réalisée sur les mâles (Denis 1978) nous permettent de conclure que :

- lorsque la vie larvaire se déroule sous une photophase moyenne (10-12 heures), les animaux entrent tous et indéfiniment en diapause.

- lorsque la vie larvaire se déroule sous une photophase longue, une partie des animaux présente un développement direct, tandis que l'autre partie subit une diapause mais limitée.

- Des résultats comparables sont obtenus lorsque la vie larvaire se déroule sous photophase courte, mais pour une partie seulement des animaux ; les autres semblent incapables de se métamorphoser.

- Les conditions critiques, comprises entre celles qui déclenchent et entretiennent indéfiniment la diapause et celles qui provoquent un développement direct ou une diapause limitée, correspondent à des photophases voisines de 14 heures, d'une part, et de 8 heures, d'autre part.

- L'échelonnement des entrées en nymphose, observé dans tous les cas, traduit une grande variabilité des réactions individuelles aux conditions expérimentales. La méthode utilisée pour savoir si un animal a eu un développement direct ou non est malgré tout imprécise et valable uniquement pour les mâles. Il serait donc illusoire de vouloir déterminer des proportions entre les larves qui ont subi une diapause et celles qui n'en ont pas subi.

- Les mâles rompent leur diapause plus aisément que les femelles.

Les réactions d'Anabolia nervosa vis-à-vis de la photopériode s'apparentent à celles d'Ostrinia nubilalis (BECK 1974). Toutefois, dans des conditions données, les réactions individuelles de $A$. nervosa sont toujours très variées.

\section{5. - CONDITIONS NECESSAIRES POUR LA RUPTURE DE LA DIAPAUSE}

Les expériences suivantes concernent des larves chez lesquelles a été induite une diapause illimitée par élevage sous photophase de 12 heures. 


\subsection{Effet d'un changement de photopériode pendant la diapause}

Nous avons vu que si des animaux étaient maintenus à la photopériode qui a conditionné la diapause, ils étaient incapables de se métamorphoser. Nous avons alors cherché à rompre cette diapause par un changement de photopériode. Pour cela, deux lots de 25 larves en diapause depuis environ un mois ont été exposés l'un à une photophase de 18 heures et l'autre à une photophase de 6 heures.

\section{RÉSUltats (Tableau II)}

Tableau II. - Effet d'un changement de photopériode sur des larves en diapause.

\begin{tabular}{ccc}
\hline Photophase & $\begin{array}{c}\text { Délai entre début exposition et entrée en nym- } \\
\text { phose }\end{array}$ & $\begin{array}{c}\text { Echelonnement } \\
\text { des entrées } \\
\text { en nymphose }\end{array}$ \\
\hline $18 \mathrm{~h} \quad \begin{array}{r}99 \text { à } 136 \text { iours et } \\
2 \text { larves mortes entre-temps }\end{array}$ & 37 jours \\
$\quad \begin{array}{l}193 \text { à } 225 \text { jours pour } 3 \text { mâles } \\
\text { - Les autres morts à l'état larvaire } 4 \text { à } 7 \text { mois } \\
\text { et demi plus tard }\end{array}$ & 32 jours \\
\hline
\end{tabular}

Sous photophase de 18 heures, les animaux rompent tous leur diapause, et cela entre un peu plus de trois mois et quatre mois et demi après le début de l'exposition. D'autres expériences réalisées sous photophase de 16 heures et de 15 heures ont fourni des résultats analogues à ceux-ci.

Sous photophase de 6 heures, la diapause peu également être rompue, mais pour quelques individus seulement, essentiellement des mâles, et après une exposition d'au moins six mois et demi.

\subsection{Durée nécessaire de l'action photopériodique pour rompre la diapause}

\section{- Photopériode à photophase longue}

Nous avons vu que la rupture de la diapause était obtenue principalement par l'action d'une photopériode de type estival. Or dans la nature, les nymphoses se produisent après diminution de la photophase. Nous avons donc cherché à déterminer le temps nécessaire d'exposition à une photopériode stimulante pour rompre la diapause. Pour cela, des lots de 25 larves en diapause ont été exposés plus ou moins longtemps à une photophase de 16 heures, puis ramenés sous photophase initiale de 12 heures. Le choix d'une photophase de 16 heu- 
res tient à ce qu'elle correspond sensiblement à la durée des périodes diurnes pendant la majeure partie des mois de juin et de juillet, aux latitudes de la France.

\section{RÉSUltats (Tableau III)}

Tableau III. - Rôle du temps d'action d'une photopériode stimulante sur la rupture de la diapause.

\begin{tabular}{|c|c|c|c|}
\hline $\begin{array}{c}\text { Durée } \\
\text { exposition } \\
\text { photophase } \\
16 \mathrm{~h}\end{array}$ & $\begin{array}{c}\text { Délai } \\
\text { entre retour } \\
\text { sous photophase } \\
12 \mathrm{~h} \text { et entrée } \\
\text { en nymphose }\end{array}$ & $\begin{array}{l}\text { Echelonnement } \\
\text { des entrées } \\
\text { en nymphose }\end{array}$ & Observations \\
\hline 7 jours & - & - & aucune nymphose \\
\hline 9 jours & - & - & aucune nymphose \\
\hline 12 jours & 82 jours & - & 1 nymphe mâle \\
\hline 15 jours & 42 à 126 jours & 84 jours & $\begin{array}{l}8 \text { nymphes dont } 6 \text { mâles et } \\
2 \text { femelles }\end{array}$ \\
\hline $1 \mathrm{mois}$ & 43 à 121 jours & 78 jours & $\begin{array}{l}14 \text { nymphes dont } \\
9 \text { mâles et } 5 \text { femelles }\end{array}$ \\
\hline 2 mois & 32 à 78 jours & 46 jours & 25 nymphes \\
\hline 100 jours & 11 à 23 jours & 12 jours & 25 nymphes \\
\hline
\end{tabular}

Il faut une exposition minimum de douze jours à la photopériode estivale pour que de rares nymphoses se produisent. Mais une exposition d'environ deux mois est nécessaire pour que tous les animaux puissent se métamorphoser. Et lorsque la durée d'exposition est supérieure, les entrées en nymphose sont très groupées. La comparaison entre la dernière ligne du tableau III et la première ligne du tableau II montre qu'après une action prolongée de la photopériode stimulante, une diminution de la photophase accélère la rupture de la diapause.

Par ailleurs dans les cas où une partie seulement des animaux rompt la diapause, il s'agit surtout de mâles. Et dans tous les cas, la majorité des mâles entre en nymphose avant la majorité des femelles.

\section{- Photopériode à photophase courte}

25 larves en diapause ont été exposées à une photophase de 6 heures durant trois mois, puis ramenées sous photophase de 12 heures. Sept individus, dont quatre mâles et trois femelles sont entrés en nymphose de un à trois mois après le transfert à 12 heures; les autres sont morts à l'état larvaire entre deux et cinq mois après ce transfert.

La comparaison entre ce résultat et celui de la dernière ligne du tableau II montre qu'après l'action d'une photopériode à photophase 
courte, une augmentation de la photophase facilite la rupture de la diapause.

\section{6. - CONCLUSION ET DISCUSSION}

Une photopériode à photophase moyenne (10-12 heures) maintenue pendant toute la vie larvaire induit à la fin de la croissance une diapause de tous les animaux et stoppe indéfiniment leur développement. Et pour que la métamorphose puisse se produire, les larves en diapause doivent être exposées à une photopériode à photophase longue (de 15 heures ou plus). Les longues périodes diurnes estivales ne sont donc pas responsables de la diapause, comme Novak (1960) le pensait. Et c'est au contraire parce que cette dernière a lieu en été que les animaux peuvent ensuite se métamorphoser.

Le tableau III montre que la photopériode stimulante doit agir pendant au moins deux mois pour que l'ensemble des animaux puisse entrer en nymphose et cela au mieux une centaine de jours plus tard. Or, dans la nature, les animaux terminent leur croissance durant la seconde quinzaine de mai ou début juin et reçoivent, aux latitudes de la France, un éclairement journalier de 15 à 16 heures pendant deux mois à deux mois et demi depuis la seconde quinzaine de mai jusqu'à fin juillet, c'est-à-dire sensiblement durant le temps de stimulation nécessaire à la reprise de leur développement.

Le tableau III montre en outre (ligne 6) que les entrées en nymphose commencent environ trois mois après le début de l'action de la photopériode stimulante et s'échelonnent pendant un mois et demi. Ceci concorde avec nos observations puisque nous avons noté les premières nymphoses peu après la mi-août et les dernières, début octobre.

Les animaux présentent un développement direct ou une courte diapause lorsque leur croissance s'est effectuée sous photophase longue (de 16 heures ou plus). Et nous avons déjà indiqué que le stade sensible à l'action de la photopériode était principalement le troisième. Des résultats comparables sont obtenus lorsque la croissance s'est effectuée sous photophase courte (inférieure à 8 heures), mais pour une partie seulement des animaux. Et nous avons vérifié que, là erıcore, le troisième stade était le stade sensible à l'action de la photopériode. Enfin lorsque la croissance s'est déroulée sous une photophase de 14 heures ou de 8 heures, quelques individus, essentiellement des mâles, peuvent entrer en nymphose après une diapause de deux mois. Or aux latitudes de la France, les périodes diurnes sont roisines de 8 heures pendant la seconde quinzaine de décembre et début janvier. Et nous avons trouvé en Bretagne des larves du troi- 
sième stade en décenıre. Ces animaux dont le développement est en avance sur celui de leurs congénères peuvent fort bien terminer leur croissance entre fin avril et début mai. Une diapause d'environ deux mois pour certains d'entre eux les ferait émerger durant la seconde quinzaine de juillet, compte tenu des trois semaines de la nymphose. Ces déductions concordent avec les données de Killington en 1930 , Baker en 1958 et Crichton en 1970. Et il est possible d'envisager que dans les régions septentrionales où la photophase est inférieure à 8 heures pendant une partie de l'hiver, certains individus puissent effectuer un cycle pratiquement sans diapause. Ainsi s'expliquerait la présence d'adultes dès le mois de juin, signalée par Brindle en 1965 au Nord de l'Angleterre.

Toutes nos expériences montrent que, d'une manière générale, les mâles rompent leur diapause plus aisément que les femelles. Ceci va dans le sens de nos données concernant les animaux de la nature dont la majorité des mâles émerge un peu avant la majorité des femelles.

Remerciements. - Nous sommes infiniment reconnaissant au $\mathrm{D}^{\mathrm{r}}$ Crichton de l'Université de Reading (Angleterre) de nous avoir si aimablement communiqué les données qu'il a recueilies sur A. nervosa et nous le remercions très vivement de nous avoir permis d'en faire état. Les travaux du $\mathrm{D}^{\mathrm{r}}$ Crichton ont été rendus possible grâce à l'aide du Rothamsed Insect Survey que nous tenons également à remercier.

\section{TRAVAUX CITES}

Beck (S. D.). 1974. - Photoperiodic determination of insect development and diapause. I. Oscillators, hourglass and a determination model. J. comp. Physiol., 90, 275-296.

Botosaneanu (L.). 1957. - Recherches sur les Trichoptères (imagos) de Roumanie. Bull. entomol. Pologne, 26, 383-433.

BrINDLE (A.). 1965. - The flight period of Trichoptera (Caddis-flies) in Northern England. Entomologist's Rec., 77, 148-159.

CRIchton (M. I.) et BAKER (B. R.). 1959. - Records of Trichoptera from the Reading area, 1957-1958. Entomologist's mon. Mag., 95, 85-87.

DeNis (C.). 1974. - Obtention d'un cycle biologique sans diapause chez Limnephilus rhombicus et Anabolia nervosa (Trichoptera, Limnephilidae). Bull. Soc. Sci. Bretagne, 48, 197-207.

Denis (C.). 1978. - Larval and imaginal diapauses inLimnephilidae. Proc. 2nd Intern. Symp. on Trichoptera, Reading, 109-115.

HanNa (H. M.), 1957. - A study of the growth and feeding habits of the larvae of four species of caddis flies. Proc. R. ent. Soc. Lond. (A), 32, 139-146.

Killingron (F. J.). 1930. - Neuroptera (sensu lato) from France, August, September 1929. Entomologist, 63, 111-112.

LE LANNIC (J.). 1976. - Développement de l'appareil reproducteur de quelques Trichoptères Limnephilides et premières données expérimentales sur son fonctionnement. Thèse $3^{e}$ Cycle, Rennes.

NovaK (K.). 1960. - Entwicklung und Diapause des Köcherfliegenlarven Anabolia furcata Br. (Trichoptera). Acta Soc. ent. Cechoslov., 57, 207-212. 